\title{
Continuous treatment of non-sterile hospital wastewater by Trametes versicolor: How to increase fungal viability by means of operational strategies and pretreatments.
}

\author{
J. A. Mir-Tutusaus ${ }^{a}$, M. Sarrà ${ }^{*}$, G. Caminal ${ }^{b}$ \\ a'Departament d'Enginyeria Química Biològica i Ambiental, Escola d'Enginyeria, \\ Universitat Autònoma de Barcelona, 08193 Bellaterra, Barcelona, Spain \\ bInstitut de Química Avançada de Catalunya (IQAC) CSIC. Jordi Girona 18-26, \\ 08034 Barcelona, Spain
}

\begin{abstract}
Hospital wastewaters have a high load of pharmaceutical active compounds (PhACs). Fungal treatments could be appropriate for source treatment of such effluents but the transition to non-sterile conditions proved to be difficult due to competition with indigenous microorganisms, resulting in very short-duration operations. In this article, coagulation-flocculation and UV-radiation processes were studied as pretreatments to a fungal reactor treating non-sterile hospital wastewater in sequential batch operation and continuous operation modes. The influent was spiked with ibuprofen and ketoprofen, and both compounds were successfully degraded by over $80 \%$. High concentrations of microorganisms seemed to reduce the activity of the fungus and therefore the longevity of the treatment. The best strategy was the addition of a coagulation-flocculation pretreatment to a continuous reactor, which led to an operation of 28 days without biomass renovation.
\end{abstract}

Keywords: fungal bioreactor, pharmaceutical active compounds, pretreatment, non-sterile, hospital wastewater

*Corresponding author: Tel +34935812789; Fax: +34935812013; e-mail address: Montserrat.Sarra@uab.cat

\section{Introduction}

There is a growing concern among regulatory agencies and in the scientific community about pharmaceutical active compounds (PhACs) occurring in environmental water bodies. Healthcare waste management is imperative but hospital wastewater (HWW), with similar pollutant load as urban wastewater but much higher concentrations of PhACs, is commonly discharged into public sewage systems [1]. HWW constitutes the main source of PhACs in the influent of wastewater treatment plants (WWTP), which are not designed to remove these pollutants [2,3]. Consequently, these effluents could be a vector to introduce PhACs in the environment through wastewater reuse purposes such 
as irrigation, landscape and surface or groundwater replenishment [4]. Therefore, a specific HWW treatment step before the HWW is mixed with urban wastewater would prevent PhAC contamination of the WWTP influent. Among the potential technologies for removing recalcitrant organic pollutants in wastewater, fungal treatment is a particularly promising strategy to biodegrade those pollutants due to their unspecific enzymatic systems. Several studies have reported the capacity of white rot fungi (and particularly Trametes versicolor) in degrading a wide range of emerging pollutants including PhACs $[5,6]$. These studies have been carried out at different scales, from within Erlenmeyer flasks to bench bioreactors, and they have been conducted mainly in sterile conditions to best monitor the fungal degradation during the batch process. The studied pollutant concentrations are on the order of a few $\mathrm{mg} \cdot \mathrm{L}^{-1}$, but the typical concentrations in wastewater are on the order of $1 \mathrm{ng} \cdot \mathrm{L}^{-1}[7,8]$.

Moreover, the long-term operation of fungal biodegradation processes during a continuous fungal treatment of a synthetic textile wastewater, in sterile conditions, was demonstrated with a cellular retention time (CRT) of 21 days [9]. The biomass was retained in the reactor but periodic partial biomass removals were performed to limit the aging of the biomass and consequently to guarantee that metabolic activity occurred under growth-limiting conditions. However, the partial biomass renovation strategy was not enough to maintain fungus viability during the treatment of HWW under non-sterile conditions [8]. Few other references can be found that investigate the treatment of non-sterile wastewater by fungi in a continuous mode. The non-favorable competition between the inoculated fungus and the microorganisms in the real wastewater demonstrated the difficulty of developing an efficient treatment on an industrial scale due to the relatively short fungal viability time [10-13].

A novel strategy to extend the fungal viability period has not yet been established and is essential to guarantee the long-term operation for the continuous treatment of HWW, which may be achieved through a continuous pumping of the influent or by sequential batch reactor (SBR) operation. In both strategies the biomass is retained in the bioreactor. In addition, a pretreatment for the HWW may reduce the bacteria level in the influent of the fungal bioreactor and consequently extend its viability. These processes include coagulation-flocculation and UV radiation, both methods widely used in WWTP $[14,15]$. Their technology can be readily applied, but none of the methods have been used as pretreatments before.

Therefore, the main objective of this study is to increase the $T$. versicolor viability inside the reactor during a continuous treatment of non-sterile HWW. To achieve this, the following two approaches have been examined: first, the addition of two pretreatments, a coagulation-flocculation step and a UV irradiation step, and second, the continuous operation of the reactor and a SBR. The influence of both strategies on the viability period has been studied. The 
HWW was spiked with ibuprofen (IBU) and ketoprofen (KETO) for analytical purposes.

\section{Materials and methods}

\subsection{Reagents, fungus and hospital wastewater}

Ketoprofen and ibuprofen were purchased from Sigma-Aldrich (Barcelona, Spain). Thiamine hydrochloride was acquired from Merck (Barcelona, Spain), peptone and yeast extract from Scharlau (Barcelona, Spain) and glucose, ammonium chloride and other chemicals were purchased from Sigma-Aldrich (Barcelona, Spain). PhACs were of HPLC purity grade (>99\%) and all other chemicals used were of analytical grade.

T. versicolor (ATCC\#42530) was maintained on $2 \%$ malt agar slants at $25^{\circ} \mathrm{C}$ until use. Subcultures were routinely made. A mycelial suspension of $T$. versicolor was obtained as previously described by Borràs et al. [16].

The HWW was collected directly from the sewer manifold of Josep Trueta Hospital (Girona, Catalonia) in the NE of Spain. Fresh samples were collected prior to every experiment and stored at $4{ }^{\circ} \mathrm{C}$. The characteristics of the wastewaters are summarized in Table 1.

\subsection{Medium and pellet formation}

Fungal pellets were obtained by inoculating $2.7 \mathrm{~mL} \cdot \mathrm{L}^{-1}$ of the mycelial suspension in a1.5 $\mathrm{L}$ of defined medium in a sterile glass air-pulsed fluidized bioreactor. The medium contained per liter: $10 \mathrm{~g}$ glucose, $100 \mathrm{~mL}$ macronutrients, $10 \mathrm{~mL}$ micronutrients, $2.1 \mathrm{~g} \mathrm{NH}_{4} \mathrm{Cl}$ and $10 \mathrm{mg}$ thiamine [16]. The $\mathrm{pH}$ was controlled at 4.5 by adding $\mathrm{HCl} 1 \mathrm{M}$ or $\mathrm{NaOH} 1 \mathrm{M}$ and the $\mathrm{O}_{2}$ was measured to ensure proper aeration. Fluidized conditions in the reactors were maintained by using $1 \mathrm{~s}$ air pulse every $4 \mathrm{~s}$. The aeration rate was $0.8 \mathrm{~L} \cdot \mathrm{min}^{-1}$ and the temperature was maintained at $25^{\circ} \mathrm{C}$.

\subsection{Coagulation-flocculation pretreatment}

The coagulation-flocculation experiments were carried out following ASTM D2035-13 guidelines [17] in ajar-test apparatus (Flocculator SW1 from Stuart Scientific, Staffordshire, UK). Coagulants HypoIDW217 and HyflocAC50 and flocculants HimolocJO2030 and HimolocDR3000 were kindly provided by Derypol, S.A. (Barcelona, Spain). The jar tests involved 2 min of coagulation at $200 \mathrm{rpm}, 15 \mathrm{~min}$ of flocculation at $20 \mathrm{rpm}$ and $30 \mathrm{~min}$ of settling.

\subsection{UV-C irradiation pretreatment}

The HWW pretreated by coagulation-flocculation was stored at room temperature in a $2 \mathrm{~L}$ sterile glass bottle and stirred magnetically. This pretreated 
HWW was then pumped, using a $0.5 \mathrm{~L} / \mathrm{D}$ flow rate, into a Jebo UV-H9 UV-C sterilizer unit ( $9 \mathrm{~W}$, mercury-arc low pressure lamp, $254 \mathrm{~nm}$ wavelength, JEBO, United States) with a residence time of $9.6 \mathrm{~h}$. The effluent was collected in a sterile bottle until the volume required to fill the bioreactor was reached (1.5 L). Samples for the heterotrophic plate count and absorbance measurement at 650 $\mathrm{nm}$ were taken every $24 \mathrm{~h}$.

\subsection{HWW treatments}

After the pellet growth, the medium was withdrawn, and the bioreactor was filled with untreated HWW or pretreated HWW, depending on the experiment. Before placement in the bioreactor, the influent was spiked with ketoprofen and ibuprofen to reach a total concentration of $20 \mathrm{mg} \cdot \mathrm{L}^{-1}$ of each PhAC. Two bioreactors were run in parallel, one operating continuously with a hydraulic residence time (HRT) of 3 days and the other operating as an SBR with a cycle of $3 \mathrm{~d}$. The first experiment used raw HWW2, the second experiment used HWW1 with a coagulation-flocculation pretreatment, and the third experiment used HWW3 with a coagulation-flocculation process followed by a UV irradiation step, as summarized in Fig. 1.

\subsection{PhACs analysis}

Samples were filtered through a Millipore Millex-GV PVDF $0.22 \mu \mathrm{m}$ membrane and placed in amber vials to avoid photodegradation. Analyses were carried out using a Dionex Ultimate 3000 HPLC system equipped with a UV detector. The separation was achieved on a GraceSmart RP 18 column $(250 \mathrm{~mm} \times 4.6 \mathrm{~mm}$, particle size $5 \mu \mathrm{m}$ ). The mobile phase consisted of $0.01 \mathrm{M} \mathrm{K}_{2} \mathrm{HPO}_{4}$ containing 2 $\mathrm{g} \cdot \mathrm{L}^{-1}$ methane sulphonic acid adjusted to $\mathrm{pH} 6.5$ (Pump A) and acetonitrile (Pump B). The flow rate was $1.5 \mathrm{~mL} \cdot \mathrm{min}^{-1}$ and the eluent gradient started at $15 \% \mathrm{~B}$ and increased to $50 \%$ from 0 to $15 \mathrm{~min}$; the gradient decreased to $15 \%$ $\mathrm{B}$ from 15 to $16 \mathrm{~min}$ and remained at $15 \% \mathrm{~B}$ from 16 to $20 \mathrm{~min}$. A sample volume of $20 \mu \mathrm{L}$ was injected from a Dionex autosampler and the detection was carried out at $210 \mathrm{~nm}$. All determinations were performed at $30^{\circ} \mathrm{C}$.

\subsection{Other analyses}

Glucose concentration was measured per triplicate with an YSI 2700 SELECT enzymatic analyzer (Yellow Spring Instruments). Laccase activity was measured per triplicate using a modified version of the method for the determination of manganese peroxidase where 2,6-dimetoxyphenol (DMP) is oxidized by laccase in the absence of a cofactor [18]. Changes in the absorbance at $468 \mathrm{~nm}$ were monitored for $2 \mathrm{~min}$ on a Varian Cary 3 UV/Vis spectrophotometer at $30^{\circ} \mathrm{C}$. Activity units per liter $\left(U \cdot \mathrm{L}^{-1}\right)$ are defined as the micromoles per liter of DMP oxidized per minute. The molar extinction coefficient of DMP was $24.8 \mathrm{mM}^{-1} \cdot \mathrm{cm}^{-1}$ [19]. The conductivity was determined by a CRISON MicroCM 2100 conductometer, and the absorbance at $650 \mathrm{~nm}$ 
was monitored by a UNICAM 8625 UV/VIS spectrometer. Heterotrophic plate count (HPC) was analyzed per triplicate according to APHA (1995) [20]. Chloride, sulfate, nitrate and phosphate anions were quantified by a Dionex ICS-2000 ionic chromatograph. The total suspended solids (TSS), dissolved inorganic carbon (DIC) and dissolved organic carbon (DOC) were determined

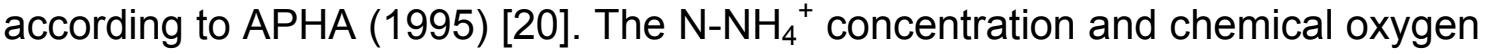
demand (COD) were analyzed by using commercial kits LCH303 and LCK114 or LCK314, respectively (Hach Lange, Germany).

\section{Results and discussion}

Three hospital wastewaters, HWW1 through HWW3, were characterized physically, chemically and biologically (Table 1). Most of the parameters are in the same range as other hospital effluents with the exception of conductivity and chloride concentration, which were higher than those reported [3,7]. Although their concentrations were not uncommonly high, the COD and TSS concentrations in HWW3 were twice that in HWW1 and HWW2, while the ammonia concentration was very low. HWW3 also had the highest chloride concentration $\left(15,723 \mathrm{mg} \mathrm{Cl} \cdot \mathrm{L}^{-1}\right)$. However, HWW1 had the greatest HPC, 4.3.1 $10^{9} \mathrm{cfu} \cdot \mathrm{mL}^{-1}$, while HWW2 and HWW3 had counts in the $10^{7} \mathrm{cfu} \cdot \mathrm{mL}^{-1}$ range.

\subsection{Study of the pretreatments}

\subsubsection{Coagulation-flocculation}

The type and dosage of the coagulants are two of the most important parameters to consider when optimizing this process [21]. HypolDW217 and HyflocAC50 were evaluated as coagulants at concentrations of $1-5 \mathrm{mg} \cdot \mathrm{L}^{-1}$ and $150-300 \mathrm{mg} \cdot \mathrm{L}^{-1}$, respectively, and HimolocJO2030 and HimolocDR3000 were evaluated as flocculants at a concentration of $15-30 \mathrm{mg} \cdot \mathrm{L}^{-1}$, as recommended by the company who provided these products. Table 2 shows the experimental design and the obtained results. Coagulant HypolDW217 produced consistently low absorbance reductions, independent of the flocculant type and concentration used, while coagulant HyflocAC50 achieved zero absorbance values with both flocculants and at almost all tested concentrations. Consequently, HyflocAC50 is considered as a suitable coagulant for reducing the absorbance of HWW. The results were consistent regardless of the flocculant used, even when taking into account that HimolocDR3000 is a high molecular weight flocculant with medium cationicity and HimolocJO2030 is a very high molecular weight flocculant with high anionicity. Experiment 15, nonetheless, showed a typical coagulant overdose behavior visible only with DR3000 $[15,21,22]$. This result suggests that the different characteristics of the two flocculants did indeed play a distinct role in the coagulation-flocculation process. Finally, products HyflocAC50 and HimolocDR3000 were chosen as the coagulant and flocculant, respectively. 
The final concentrations of the coagulant and flocculant had to be adjusted for every HWW. Table 3 summarizes the doses used in each pretreatment, while Table 1 shows the physical, chemical and biological characterization of the three raw and pretreated wastewaters used. In general, pretreated samples have similar or lower parameters than the raw wastewaters [23]. The HPC reduction achieved after the coagulation-flocculation pretreatment is very important, with reductions by factors of 10 to $10^{4}$. Additionally, a substantial reduction in TSS is observed in all 3 cases, although a reduction in COD is only observed in 2 out of $3 \mathrm{HWWs}$ tested.

\subsubsection{UV-C irradiation}

UV disinfection is a widely known technology used as a tertiary treatment in WWTP as an attractive alternative to chlorination/dechlorination processes because it is a chemical-free process, reduces the presence of potential chlorinated hydrocarbons and also deactivates chlorine-resistant microorganisms $[24,25]$. However, its use as a pretreatment is much less studied. The experiments were planned as preliminary work to determine whether UV-C irradiation could be a suitable pretreatment for the bioreactor influent.

Several studies highlight the role of the concentration and size of the suspended solids in UV disinfection $[25,26]$. Particles provide shelter to microorganisms against UV by absorbing or reflecting the UV. This can be partially overcome by using higher intensities and contact times, although the improvement is still limited. Das [24] proposed $5-10 \mathrm{mg} \cdot \mathrm{L}^{-1}$ of TSS as an acceptable range in which UV disinfection is effective. The TSS of the raw HWW used in the study ranged from $145-350 \mathrm{mg} \cdot \mathrm{L}^{-1}$, which is much higher than the maximum of that acceptable range and could thus explain the ineffectiveness of the UV treatment. However, the flocculated HWWs had a TSS level of $30-105 \mathrm{mg} \cdot \mathrm{L}^{-1}$, still above the recommended range. Nevertheless, a 10 to 100 - fold diminution of HPC was obtained. This may be explained by the long contact time used in the experiments. No significant reduction in absorbance was found, due to the flocculated HWW having values very close to zero. Therefore, UV-C irradiation was applied as a pretreatment only after a coagulation-flocculation process.

\subsection{Bioreactor operation}

The testing of both the HWW pretreatment and the operation mode was performed to find approaches that increase the duration of Trametes versicolor viability in the bioreactor. Although widely used in wastewater and drinking water treatment plants, coagulation-flocculation and UV-radiation had not been studied as pretreatments for a fungal operation. As summarized in Fig. 1, three experiments were conducted with (A) no pretreatment, (B) coagulationflocculation pretreatment and $(C)$ coagulation-flocculation followed by a UV- 
radiation pretreatment. In each experiment, two reactors were operated simultaneously: one in continuous mode (1) and one in SBR mode (2). As reviewed elsewhere, the SBR operation would act as selective disinfection, namely, the elimination of undesired microorganisms while retaining the fungal biomass [27]. Both continuous and SBR modes had an HRT of $3 \mathrm{~d}$, as the processes of pellet settling and liquid phase draining and refilling in the SBR took only $15 \mathrm{~min}$ and $95 \%$ of the liquid could be removed. Glucose and $\mathrm{NH}_{4} \mathrm{Cl}$ were added at their consumption rates to keep the fungus in growth-limiting conditions and to prevent COD increases in the effluent. A low carbon-tonitrogen ratio of 7.5 was chosen to enhance the fungus survival over the native bacteria [28].

HPC, laccase activity, and IBU and KETO removal were monitored; the results are presented in Fig. 2-4. The operation was stopped after repeated measures of the IBU and KETO degradation percentage dropped below $80 \%$.

\subsubsection{Heterotrophic plate count evolution}

Most of the studies found in the literature focus on degradation of selected compounds in sterile conditions or non-sterile tap water $[6,12,29,30]$. However, Espinosa-Ortiz et al. (2016) highlighted the necessity of working in non-sterile matrices [27]. In real non-sterile hospital wastewater, concentration of microorganisms, expressed as HPC, have special importance. Fig. 2 shows the HPC results for all the experiments. In experiment $A$, with no pretreatment, both the continuous operation and the SBR exhibited a 100-fold increase in the initial concentration of heterotrophs after $3 \mathrm{~d}$ of treatment, with an HPC of approximately $10^{8} \mathrm{cfu} \cdot \mathrm{mL}^{-1}$ after $6 \mathrm{~d}$ operation. Experiment $\mathrm{B}$, with coagulationflocculation pretreatment, exhibited a slight increase in the continuous treatment during the $27 \mathrm{~d}$ of treatment, which leads to a 100-fold HPC increase by the end of the process. However, increases by factors of 10-1000 were observed in each $3 \mathrm{~d}$ SBR cycle. The dramatic change in HPC can be explained by the native $\mathrm{HHW}$ microorganisms consuming the glucose and $\mathrm{NH}_{4} \mathrm{Cl}$ added in addition to the nutrients in the wastewater itself. The resulting heterotroph population could be adhered to the fungal biomass and suspended solids. The final HPC was approximately $10^{7} \mathrm{cfu} \cdot \mathrm{mL}^{-1}$ for the continuous treatment and approximately $10^{8} \mathrm{cfu} \cdot \mathrm{mL}^{-1}$ for the SBR. In experiment C (Fig. 2C), with a coagulation-flocculation pretreatment followed by a UV radiation step, the initial concentration of heterotrophic microorganisms was lower than the other two experiments (approximately $10^{4} \mathrm{cfu} \cdot \mathrm{mL}^{-1}$ ); however, a very rapid 1000 -fold increase was detected within the first 10 days of operation in both treatment strategies. This low initial HPC was due to the UV-radiation step, while the fast increase could be either due to the regrowth of microorganisms sheltered by suspended solids or to growth of resistant bacteria due to decreased competition with other bacteria; many of which were eliminated by the UV radiation [31]. The HPC increased until it reached a concentration of $10^{8} \mathrm{cfu} \cdot \mathrm{mL}^{-}$ 
1 in the $10^{\text {th }}$ day of continuous operation but stabilized at approximately $10^{6}$ $\mathrm{cfu} \cdot \mathrm{mL}^{-1}$. The HPC in the SBR peaked at $10^{9} \mathrm{cfu} \cdot \mathrm{mL}^{-1}$ in the $4^{\text {th }}$ cycle and remained at $10^{8} \mathrm{cfu} \cdot \mathrm{mL}^{-1}$ in the last batch. There seems to be a threshold HPC of approximately $10^{8} \mathrm{cfu} \cdot \mathrm{mL}^{-1}$, beyond which the operation could not continue, regardless of the pretreatment or operation mode. In addition, the continuous mode exhibited a consistently lower HPC than that of the SBR mode.

\subsubsection{Laccase activity profile}

Fig. 3 shows the laccase profiles measured in all three experiments. Experiment A exhibited a maximum activity (Fig. $3 \mathrm{~A}$ ) of approximately $50 \mathrm{UA} \cdot \mathrm{L}^{-1}$ at Day 4 for both operation modes. The laccase profile diminished from that day up to undetectable levels after $9 \mathrm{~d}$. In experiment $\mathrm{B}$, laccase peaked at the end of the first cycle but then dropped significantly after the second cycle (Fig. 3B). In subsequent cycles, the laccase production was recovered up to the initial cycle level during the $5^{\text {th }}$ cycle; afterward, the laccase activity showed an important reduction following each additional cycle. However, low laccase production was observed in the continuous reactor. Even when the measured laccase activity decreased, it is evident that $T$. versicolor was able to produce enzymes because the laccase profile stayed above the theoretical laccase activity assuming zero production, shown as dotted lines in the stages in Fig. 3. Finally, in experiment $\mathrm{C}$, the laccase profiles (Fig. $3 \mathrm{C}$ ) showed a maximum peak at Day 6 for the continuous treatment or $2^{\text {nd }}$ batch for the SBR. Afterward, the laccase production decreased dramatically, and no laccase activity was detected after the $4^{\text {th }}$ batch or in the $14^{\text {th }}$ day of continuous operation. It is noteworthy that in experiment $\mathrm{C}$, the theoretical laccase activity assuming no production was higher than the actual activity in the reactor, indicating deactivation of the enzyme. This may be explained as an effect of bacterial competition, which has been reported to inhibit fungal enzyme secretion capacity and to inactivate the secreted enzyme $[32,33]$.

Although laccase can be produced by both fungi and bacteria [34], the control experiments with HWW demonstrated that the production of laccase by its native bacterial microorganisms was not significant (data not shown). Consequently, the laccase production may be confidently linked to fungus activity. However, low or nonexistent laccase activity cannot be the sole indicator of $T$. versicolor inactivity. Previous studies have detected the presence of the fungus with denaturing gradient gel electrophoresis (DGGE) in continuous treatments where laccase activity was very low and HWW could inhibit the laccase activity assay [10]. Several other continuous-mode fungal water treatment operations have reported very low or no extracellular enzymatic activity $[12,35]$.

3.2.3. Ibuprofen and ketoprofen degradation 
Ibuprofen and ketoprofen were the PhACs selected to spike the HWW. Both pharmaceuticals are among the most widely used anti-inflammatory drugs, and both can be degraded by $T$. versicolor $[6,36,37]$.

The results for the rates of IBU and KETO degradation are presented in Fig. 4. In experiment $A$, in the continuous treatment, $90 \%$ of ibuprofen was degraded, while ketoprofen degradation rates were below $70 \%$ after day 5 . In the SBR mode, both IBU and KETO had degradation rates of $80 \%$ and above for the first and third batches. The experiment was finally stopped at day 12 when the threshold degradation was not reached, and the breakdown of the fungal pellets into free mycelium was observed. In experiment $B$, the elimination rates of both pharmaceuticals (Fig. 4B) stayed above $80 \%$ for the majority of the continuous operation. During the SBR operation, KETO degradation was approximately $90 \%$ during the first 5 cycles but decreased significantly thereafter. However, IBU was completely degraded during 7 cycles and only was detected in the last cycle. Both reactors were operated for 28 days until the degradation rates fell below $80 \%$. Other signals of low fungal activity were also observed, including low laccase production and the loss of original biomass pellet shape. In experiment $\mathrm{C}$, the degradation of IBU remained constant at $78 \%$ during the first 14 days of continuous treatment but then increased up to $95 \%$. KETO degradation, however, was higher than $80 \%$ until Day 11 when it quickly dropped to degradation rates lower than $40 \%$. During SBR operation, KETO degradation was higher than $80 \%$ during all cycles, but the removal of KETO removal was high only in the three first cycles. At the end of fourth cycle, the degradation was minimal although it recovered to approximately $65 \%$ in the fifth cycle, when the experiment was stopped due to low fungal activity. Few references can be found regarding IBU and KETO removal in wastewaters. Badia-Fabregat et al. (2015) reported a $67 \%$ and $46 \%$ removal of IBU and KETO, respectively in sterile non-spiked urban wastewater, lower than the $80 \%$ threshold used in the present manuscript [8]. However, Nguyen et al. (2013) reported a very high $90 \%$ removal of ketoprofen in a fungus-augmented membrane bioreactor, although in sterile conditions [30].

The results of experiments $A$ and $B$ indicated that ibuprofen was eliminated faster than ketoprofen during long-term operation in both continuous and SBR strategies, although the results of experiment $C$ are slightly different. These results agree with previous reports $[5,8]$. The pathway of fungal degradation of IBU remains unknown, but it does not involve laccase. This result is in agreement with the present results, as IBU was removed when no laccase activity was detected. This pathway does not involve the cytochrome P450 system either. The UV pretreatment seemed to decrease the removal of IBU during the early reactor operation, which is possibly related to its distinct degradation pathway. Ketoprofen was eliminated in both reactors; this is consistent with previous reports, which state that KETO is degraded by $T$. 
versicolor, with the P450 system involved in the degradation and the laccase system playing only a minor role [37]. As a result, ketoprofen was degraded even when no laccase activity was detected in the bioreactor. To conclude, IBU and KETO have been effectively eliminated in both reactors when treating nonsterile HWW with long-duration treatments.

Experiment B, which used a coagulation-flocculation pretreatment before the fungal reactor, was selected as the best strategy because it exhibited the longest viability period of $28 \mathrm{~d}$. This viability period could be further extended by implementing a partial biomass renovation strategy to overcome the biomass aging process. The sequential batch reactor operations showed better IBU and KETO reductions than continuous operations in experiments $A$ and $C$ but not in experiment $B$, where the continuous operation maintained elimination between $70-90 \%$ for a long period of time. As a result, a second step in the pretreatment of HWW effluent did not improve the fungal bioreactor. In addition, the use of the continuous operation mode was chosen as the best strategy for long-term fungal degradation with fungal biomass retained in the bioreactor. This strategy has operational advantages, such as requiring less labor because it is easy to automate. To the best of our knowledge, this is the first time that an air-pulsed fungal reactor without biomass renovation treated non-sterile HWW with high elimination yields for 28 days.

\section{Conclusions}

Pretreating HWW with a coagulation-flocculation process can reduce the initial HPC level up to $10^{4} \mathrm{cfu} \cdot \mathrm{mL}^{-1}$, which extended the fungal viability during the continuous treatment of a non-sterile real effluent. The addition of a UV pretreatment did not lead to better performance of the fungal bioreactor in terms of higher PhAC reductions or longer operation time. Testing of the operation modes indicated that continuous operation is preferred over SBR because the reduction capacity of $T$. versicolor was maintained for a longer period. The optimal treatment setup, a coagulation-flocculation pretreatment coupled to a fungal treatment in an air-pulsed fluidized bioreactor with retained biomass in pellet form, maintained fungal activity for 28 days without purging or biomass renovation. Consequently, a length of operation similar to the operation in sterile conditions was achieved. Future work should consider partial biomass renovation to further extend the viable duration of fungal degradation and to overcome the biomass aging process, which are essential requirements to guarantee longer-term continuous HWW treatment.

\section{Acknowledgements}

This work has been funded by the Spanish Ministry of Economy and Competitiveness (project CTM 2013-48545-C2-1-R) and partly supported by the Generalitat de Catalunya (Consolidated Research Group 2014-SGR-0476). The Department of Chemical Engineering of UAB is member of the Xarxa de 
Referència en Biotecnologia de la Generalitat de Catalunya. J. A. Mir-Tutusaus acknowledges the predoctoral grant from UAB.

This is the author's version of a work that was accepted for publication in Journal of hazardous materials (Ed. Elsevier). Changes resulting from the publishing process, such as peer review, editing, corrections, structural formatting, and other quality control mechanisms may not be reflected in this document. Changes may have been made to this work since it was submitted for publication. A definitive version was subsequently published in Mir-Tutusaus, JA, Sarrà, M. and Caminal, G. "Continuous treatment of non-sterile hospital wastewater by Trametes versicolor: how to increase fungal viability by means of operational strategies and pretreatments" in Journal of hazardous materials, vol. 318 (Nov. 2016), p. 561-570. DOI 10.1016/j.hazmat.2016.07.036 
[1] H.A. Kamitsou, I.K. Kalavrouziotis, M. Papadaki, Healthcare waste management in the prefectures of Aetoloakarnania and the islant of Lesbos, Greece, 17 (2015) 312-322.

[2] O. Frédéric, P. Yves, Pharmaceuticals in hospital wastewater: their ecotoxicity and contribution to the environmental hazard of the effluent., Chemosphere. 115 (2014) 31-9. doi:10.1016/j.chemosphere.2014.01.016.

[3] P. Verlicchi, M. Al Aukidy, E. Zambello, What have we learned from worldwide experiences on the management and treatment of hospital effluent? - An overview and a discussion on perspectives, Sci. Total Environ. 514 (2015) 467-491. doi:10.1016/j.scitotenv.2015.02.020.

[4] D. Fatta-Kassinos, I.K. Kalavrouziotis, P.H. Koukoulakis, M.I. Vasquez, The risks associated with wastewater reuse and xenobiotics in the agroecological environment, Sci. Total Environ. 409 (2011) 3555-3563. doi:10.1016/j.scitotenv.2010.03.036.

[5] L.N. Nguyen, F.I. Hai, S. Yang, J. Kang, F.D.L. Leusch, F. Roddick, et al., Removal of pharmaceuticals, steroid hormones, phytoestrogens, UVfilters, industrial chemicals and pesticides by Trametes versicolor: Role of biosorption and biodegradation, Int. Biodeterior. Biodegrad. 88 (2014) 169-175. doi:10.1016/j.ibiod.2013.12.017.

[6] E. Marco-Urrea, M. Pérez-Trujillo, T. Vicent, G. Caminal, Ability of whiterot fungi to remove selected pharmaceuticals and identification of degradation products of ibuprofen by Trametes versicolor, Chemosphere. 74 (2009) 765-772. doi:10.1016/j.chemosphere.2008.10.040.

[7] C. Cruz-Morató, D. Lucas, M. Llorca, S. Rodriguez-Mozaz, M. Gorga, M. Petrović, et al., Hospital wastewater treatment by fungal bioreactor:

Removal efficiency for pharmaceuticals and endocrine disruptor compounds., Sci. Total Environ. 493C (2014) 365-376.

doi:10.1016/j.scitotenv.2014.05.117.

[8] M. Badia-Fabregat, D. Lucas, M. Gros, S. Rodríguez-Mozaz, D. Barceló, G. Caminal, et al., Identification of some factors affecting pharmaceutical active compounds (PhACs) removal in real wastewater. Case study of fungal treatment of reverse osmosis concentrate., J. Hazard. Mater. 283 (2015) 663-71. doi:10.1016/j.jhazmat.2014.10.007.

[9] P. Blánquez, M. Sarrà, M.T. Vicent, Study of the cellular retention time and the partial biomass renovation in a fungal decolourisation continuous process., Water Res. 40 (2006) 1650-6.

doi:10.1016/j.watres.2006.02.010.

[10] M. Badia-Fabregat, D. Lucas, M.A. Pereira, M. Alves, T. Pennanen, H. Fritze, et al., Continuous fungal treatment of non-sterile veterinary hospital effluent: pharmaceuticals removal and microbial community assessment., Appl. Microbiol. Biotechnol. (2015). doi:10.1007/s00253015-7105-0.

[11] C. Cruz-Morató, L. Ferrando-Climent, S. Rodriguez-Mozaz, D. Barceló, E. Marco-Urrea, T. Vicent, et al., Degradation of pharmaceuticals in nonsterile urban wastewater by Trametes versicolor in a fluidized bed bioreactor., Water Res. 47 (2013) 5200-10. 
doi:10.1016/j.watres.2013.06.007.

[12] S. Yang, F.I. Hai, L.D. Nghiem, L.N. Nguyen, F. Roddick, W.E. Price, Removal of bisphenol $A$ and diclofenac by a novel fungal membrane bioreactor operated under non-sterile conditions, Int. Biodeterior. Biodegrad. 85 (2013) 483-490. doi:10.1016/j.ibiod.2013.03.012.

[13] Y. Zhang, S.U. Geißen, Elimination of carbamazepine in a non-sterile fungal bioreactor, Bioresour. Technol. 112 (2012) 221-227. doi:10.1016/j.biortech.2012.02.073.

[14] E. Tzfati, M. Sein, A. Rubinov, A. Raveh, A. Bick, Pretreatment of wastewater: Optimal coagulant selection using Partial Order Scaling Analysis (POSA), J. Hazard. Mater. 190 (2011) 51-59. doi:10.1016/j.jhazmat.2011.02.023.

[15] E.A. López-Maldonado, M.T. Oropeza-Guzman, J.L. Jurado-Baizaval, A. Ochoa-Terán, Coagulation-flocculation mechanisms in wastewater treatment plants through zeta potential measurements., J. Hazard. Mater. 279 (2014) 1-10. doi:10.1016/j.jhazmat.2014.06.025.

[16] E. Borràs, P. Blánquez, M. Sarrà, G. Caminal, T. Vicent, Trametes versicolor pellets production: Low-cost medium and scale-up, Biochem. Eng. J. 42 (2008) 61-66. doi:10.1016/j.bej.2008.05.014.

[17] ASTM International, ASTM D2035-13. Standard Practice for CoagulationFlocculation Jar Test of Water, Am. 11.01 (2013) 13-16. doi:10.1520/D2035-13.2.

[18] E.E. Kaal, E. de Jong, J.A. Field, Stimulation of Ligninolytic Peroxidase Activity by Nitrogen Nutrients in the White Rot Fungus Bjerkandera sp. Strain BOS55., Appl. Environ. Microbiol. 59 (1993) 4031-6. http://www.pubmedcentral.nih.gov/articlerender.fcgi?artid=195863\&tool=p mcentrez\&rendertype=abstract (accessed January 8, 2016).

[19] H. Wariishi, K. Valli, M.H. Gold, Manganese(II) oxidation by manganese peroxidase from the basidiomycete Phanerochaete chrysosporium: Kinetic mechanism and role of chelators, J. Biol. Chem. 267 (1992) 23688-23695.

[20] APHA-AWWA-WEF, Standard Methods for the Examination of Water and Wastewater, 19th ed., American Public Association/AmericaWaterWorks Association/Water Environment Federation, Washington, 1995.

[21] V. Saritha, N. Srinivas, N. V. Srikanth Vuppala, Analysis and optimization of coagulation and flocculation process, Appl. Water Sci. (2015). doi:10.1007/s13201-014-0262-y.

[22] Y.X. Zhao, Y. Wang, B.Y. Gao, H.K. Shon, J.H. Kim, Q.Y. Yue, Coagulation performance evaluation of sodium alginate used as coagulant aid with aluminum sulfate, iron chloride and titanium tetrachloride, Desalination. 299 (2012) 79-88. doi:10.1016/j.desal.2012.05.026.

[23] X. Liu, X.-M. Li, Q. Yang, X. Yue, T.-T. Shen, W. Zheng, et al., Landfill leachate pretreatment by coagulation-flocculation process using ironbased coagulants: Optimization by response surface methodology, Chem. Eng. J. 200-202 (2012) 39-51. doi:10.1016/j.cej.2012.06.012. 
[24] Tapas K. Das, Ultraviolet disinfection application to a wastewater treatment plant, Clean Prod. Process. 3 (2001) 69-80.

doi:10.1007/s100980100108.

[25] W.A.M. Hijnen, E.F. Beerendonk, G.J. Medema, Inactivation credit of UV radiation for viruses, bacteria and protozoan (oo)cysts in water: a review., Water Res. 40 (2006) 3-22. doi:10.1016/j.watres.2005.10.030.

[26] J. Wang, L. Wang, B. Wang, J. Zhang, Q. Zou, Impact of suspended particles and enhancement techniques on ultraviolet disinfection of a secondary effluent, J. Ocean Univ. China. 5 (2006) 381-386. doi:10.1007/s11802-006-0033-y.

[27] E.J. Espinosa-Ortiz, E.R. Rene, K. Pakshirajan, E.D. van Hullebusch, P.N.L. Lens, Fungal pelleted reactors in wastewater treatment: applications and perspectives, Chem. Eng. J. 283 (2015) 553-571. doi:10.1016/j.cej.2015.07.068.

[28] J. Rousk, E. Bååth, Fungal and bacterial growth in soil with plant materials of different C/N ratios, FEMS Microbiol. Ecol. 62 (2007) 258267. doi:10.1111/j.1574-6941.2007.00398.x.

[29] J.A. Mir-Tutusaus, M. Massís-Mora, C. Corcellas, E. Eljarrat, D. Barceló, M. Sarrà, et al., Degradation of selected agrochemicals by the white rot fungus Trametes versicolor, Sci. Total Environ. 500-501 (2014) 235-242. doi:10.1016/j.scitotenv.2014.08.116.

[30] L.N. Nguyen, F.I. Hai, S. Yang, J. Kang, F.D.L. Leusch, F. Roddick, et al., Removal of trace organic contaminants by an MBR comprising a mixed culture of bacteria and white-rot fungi, Bioresour. Technol. 148 (2013) 234-241. doi:10.1016/j.biortech.2013.08.142.

[31] O.-M. Lee, H.Y. Kim, W. Park, T.-H. Kim, S. Yu, A comparative study of disinfection efficiency and regrowth control of microorganism in secondary wastewater effluent using UV, ozone, and ionizing irradiation process., J. Hazard. Mater. 295 (2015) 201-8. doi:10.1016/j.jhazmat.2015.04.016.

[32] J.A. Libra, M. Borchert, S. Banit, Competition strategies for the decolorization of a textile-reactive dye with the white-rot fungi Trametes versicolor under non-sterile conditions, Biotechnol. Bioeng. 82 (2003) 736-744. doi:10.1002/bit.10623.

[33] A. Heinfling, M.J. Martínez, A.T. Martínez, M. Bergbauer, U. Szewzyk, Transformation of industrial dyes by manganese peroxidases from Bjerkandera adusta and Pleurotus eryngii in a manganese-independent reaction, Appl. Environ. Microbiol. 64 (1998) 2788-2793. http://www.scopus.com/inward/record.url?eid=2-s2.00344734070\&partnerID=tZOtx3y1.

[34] L. Gianfreda, F. Xu, J.-M. Bollag, Laccases: A Useful Group of Oxidoreductive Enzymes, Bioremediat. J. 3 (1999) 1-26. doi:10.1080/10889869991219163.

[35] D. Gao, Y. Zeng, X. Wen, Y. Qian, Competition strategies for the incubation of white rot fungi under non-sterile conditions, Process Biochem. 43 (2008) 937-944. doi:10.1016/j.procbio.2008.04.026.

[36] N.H. Tran, T. Urase, O. Kusakabe, Biodegradation characteristics of 
pharmaceutical substances by whole fungal culture Trametes versicolor and its laccase, J. Water Environ. Technol. 8 (2010) 125-140.

doi:10.2965/jwet.2010.125.

[37] E. Marco-Urrea, M. Pérez-Trujillo, C. Cruz-Morató, G. Caminal, T. Vicent, White-rot fungus-mediated degradation of the analgesic ketoprofen and identification of intermediates by HPLC-DAD-MS and NMR., Chemosphere. 78 (2010) 474-81.

doi:10.1016/j.chemosphere.2009.10.009. 
A

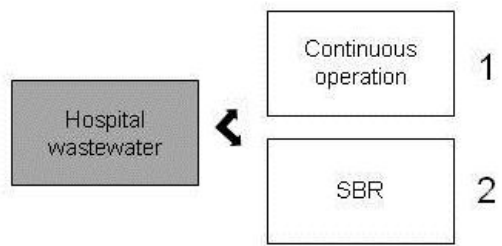

B

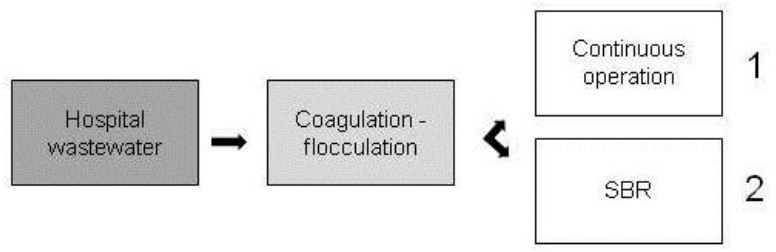

C

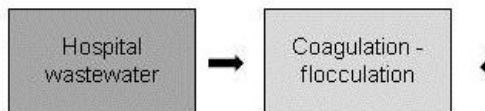

UV irradiation

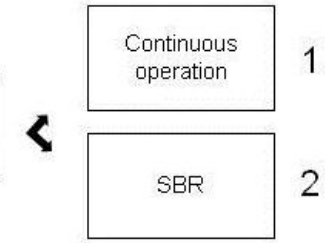

Fig. 1. Bioreactors experiments diagram. A, B and C are the experiment ID; 1 and 2 represent continuous operation and sequential batch reactor operation, respectively. Dark grey rectangles represent the initial wastewater; light grey rectangles, the pretreatment steps; white rectangles, the mode of operation. 

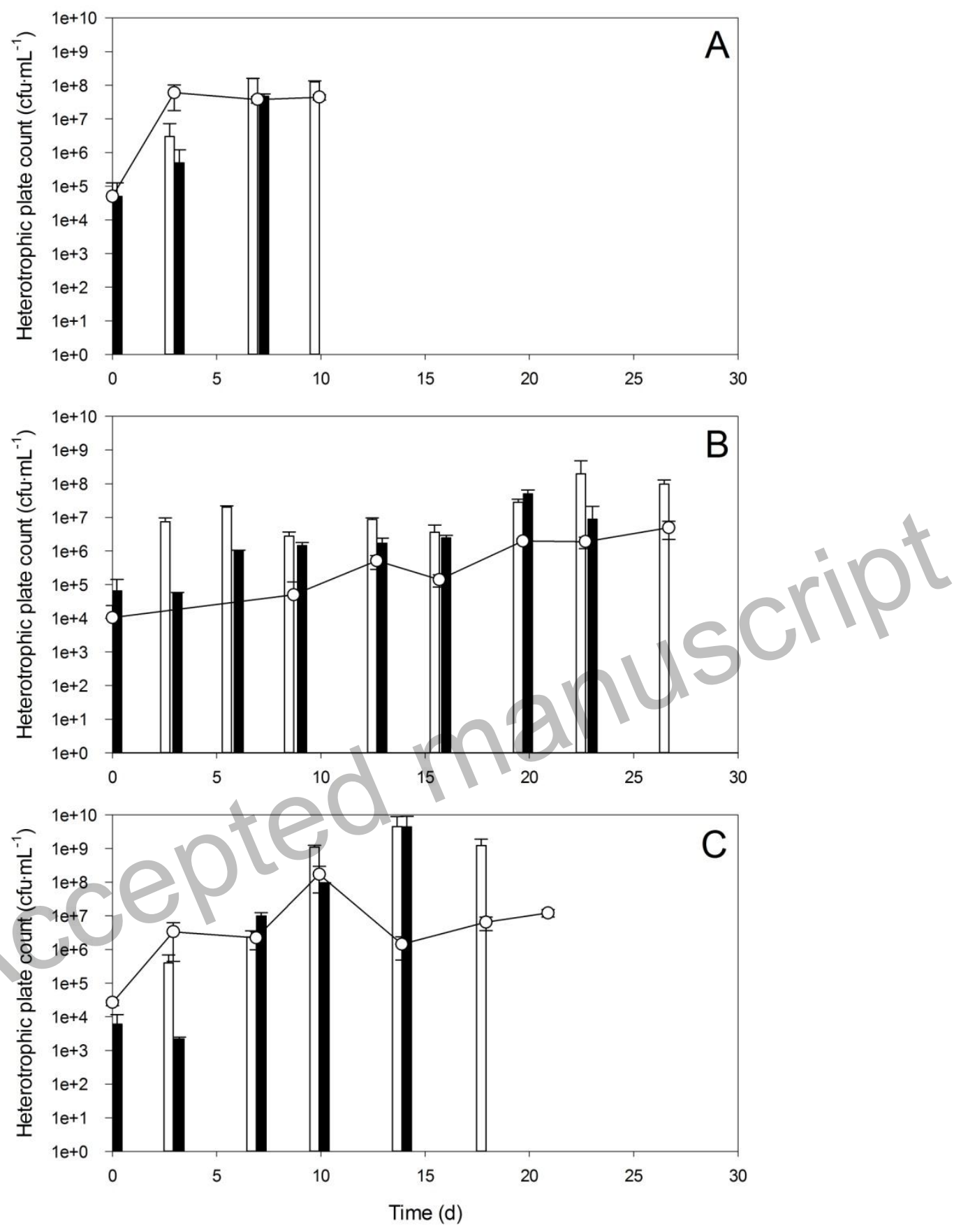

Fig. 2. Heterotrophic plate count profile in the reactors with no pretreatment $(A)$; with a flocculation step (B); with a flocculation and a UV-radiation step (C). Legend: black bars represent the initial concentration in the SBR treatment; white bars, the final concentration in the SBR treatment; white circles, the concentration profile in the continuous treatment. 

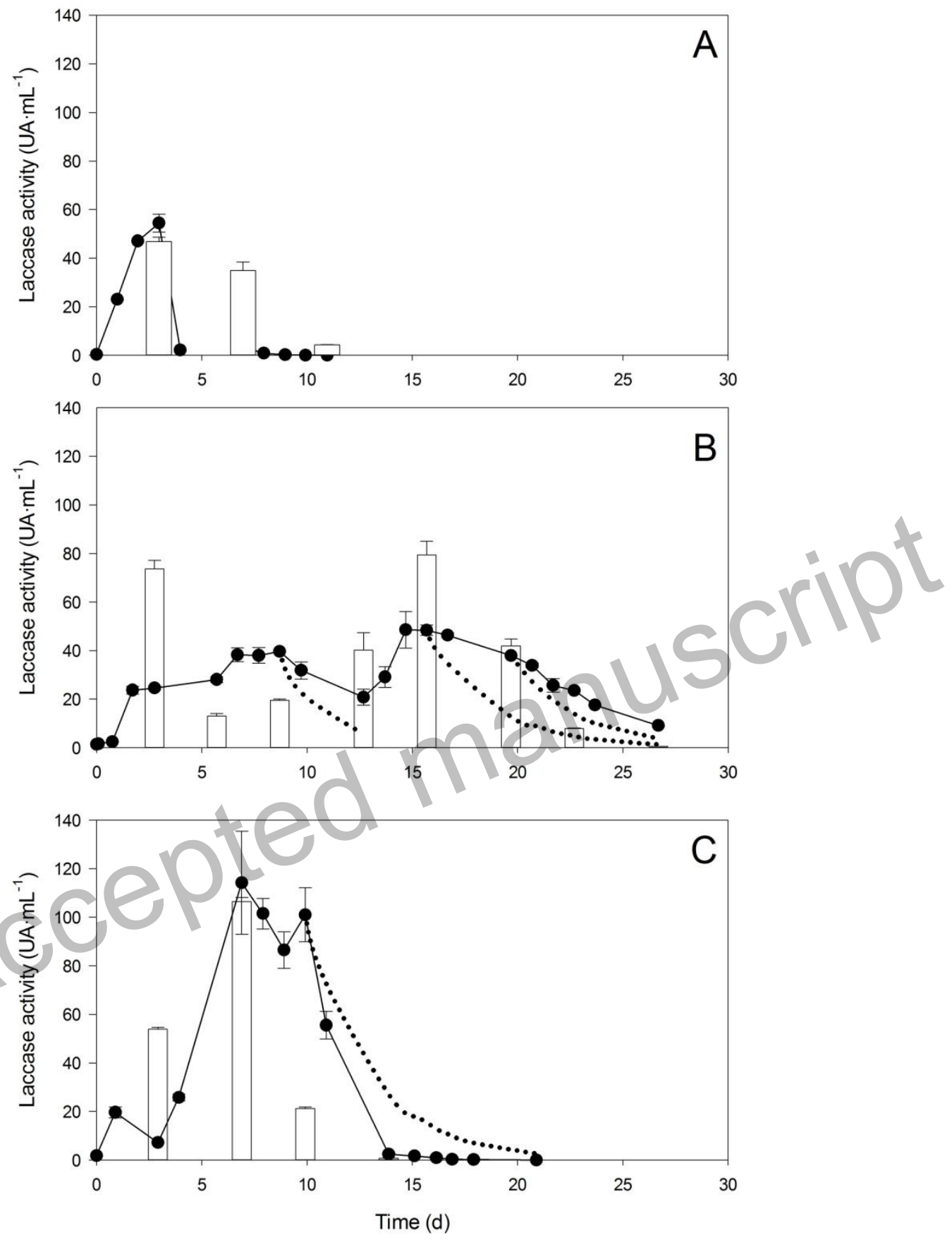

Fig. 3. Laccase activity profile in the reactors with no pretreatment (A); with a flocculation step (B); with a flocculation and a UV-radiation step (C). Legend: white bars represent the final concentration in the SBR treatment; black circles, the activity profile in the continuous treatment; dotted lines represent the theoretical concentration of laccase if no generation is assumed. 

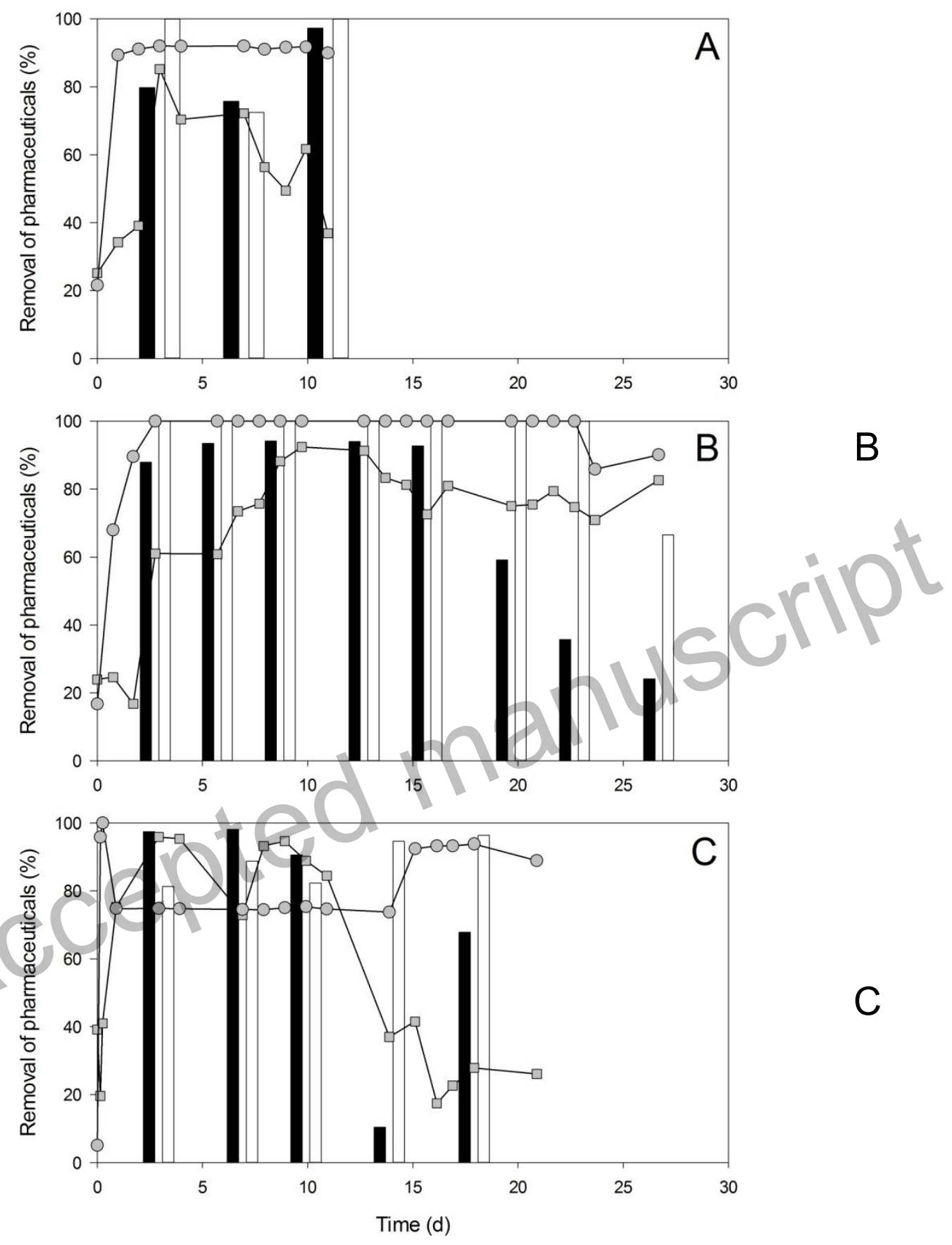

Fig. 4. Elimination profile of ketoprofen and ibuprofen in the reactors with no pretreatment $(\mathrm{A})$; with a flocculation step (B); with a flocculation and a UV-radiation step (C). Legend: black bars and white bars represent the ketoprofen and ibuprofen degradation, respectively, in the SBR; gray squares and gray circles represent the ketoprofen and ibuprofen degradation, respectively, in the continuous treatment. 
Table 1.Physical, chemical and biological characterization of hospital wastewater.

\begin{tabular}{|c|c|c|c|c|c|c|c|}
\hline Sampling date & & $22 / 01 / 2015$ & & $04 / 03 / 2015$ & & & $12 / 06 / 2015$ \\
\hline Experiment & & HWW1 & & HWW2 & & & HWW3 \\
\hline & Raw & Flocculated & Raw & Flocculated & Raw & Flocculated & Flocculated \& UV \\
\hline $\mathrm{pH}$ & 6.84 & 8.12 & 7.07 & 7.08 & 7.2 & 7.3 & 7.93 \\
\hline Conductivity $\left(\mathrm{mS} \cdot \mathrm{cm}^{-1}\right)$ & 1.23 & 1.16 & 1.72 & 1.82 & 4.69 & 4.76 & 4.46 \\
\hline Absorbance at $650 \mathrm{~nm}$ & 0.189 & 0,000 & 0.154 & 0.012 & 0.353 & 0.006 & 0.009 \\
\hline $\mathrm{HPC}\left(\mathrm{cfu} \cdot \mathrm{mL}^{-1}\right)$ & $4.3 \cdot 10^{9} \pm 3.5 \cdot 10^{8}$ & $7.5 \cdot 10^{5} \pm 2.2 \cdot 10^{5}$ & $7.4 \cdot 10^{7} \pm 1.7 \cdot 10^{7}$ & $5.4 \cdot 10^{4} \pm 7.0 \cdot 10^{3}$ & $1.9 \cdot 10^{7} \pm 6.1 \cdot 10^{6}$ & $3 \cdot 10^{6} \pm 5.2 \cdot 10^{5}$ & $4.0 \cdot 10^{5} \pm 2.9 \cdot 10^{5}$ \\
\hline Chloride (mg Cl. $\left.\mathrm{L}^{-1}\right)$ & 1603,8 & 1810,6 & 1874,0 & 2073,4 & 15723,7 & 16125,9 & 15372,9 \\
\hline Sulfate $\left(\mathrm{mg} \mathrm{S} \cdot \mathrm{L}^{-1}\right)$ & 31,6 & 25,6 & 42,0 & 37,5 & 19,8 & 25,9 & 23,4 \\
\hline Nitrate $\left(\mathrm{mg} \mathrm{N} \cdot \mathrm{L}^{-1}\right)$ & 170,6 & 190,1 & 239,9 & 223,6 & 236,7 & 287,3 & 282,8 \\
\hline Phosphate (mg P. $\left.\mathrm{L}^{-1}\right)$ & 0,2 & 0,5 & 0,1 & 0,1 & 0,7 & 0,0 & 0,2 \\
\hline Ammonia $\left(\mathrm{mg} \mathrm{N} \cdot \mathrm{L}^{-1}\right)$ & 15,5 & 8,0 & 24,4 & 8,5 & 0,2 & 1,8 & 0,1 \\
\hline $\operatorname{TSS}\left(\mathrm{mg} \cdot \mathrm{L}^{-1}\right)$ & 150 & 30 & 145 & 55 & 350 & 105 & 55 \\
\hline $\operatorname{COD}\left(\mathrm{mg} \mathrm{O}_{2} \cdot \mathrm{L}^{-1}\right)$ & $343 \pm 13$ & $108 \pm 4$ & $293 \pm 15$ & $297 \pm 1$ & $614 \pm 20$ & $313 \pm 2$ & $44 \pm 3$ \\
\hline $\operatorname{DIC}\left(\mathrm{mg} \cdot \mathrm{L}^{-1}\right)$ & $77 \pm 3$ & $63 \pm 3$ & $117 \pm 3$ & $95 \pm 1$ & $76 \pm 3$ & $73 \pm 2$ & $80 \pm 2$ \\
\hline $\mathrm{DOC}\left(\mathrm{mg} \cdot \mathrm{L}^{-1}\right)$ & $49 \pm 7$ & $47 \pm 3$ & $56 \pm 3$ & $87 \pm 2$ & $99 \pm 9$ & $90 \pm 6$ & $21 \pm 4$ \\
\hline
\end{tabular}


Table 2.Absorbance reduction of different coagulant-flocculant mixtures. Absorbance of the initial raw wastewater was 0.189 .

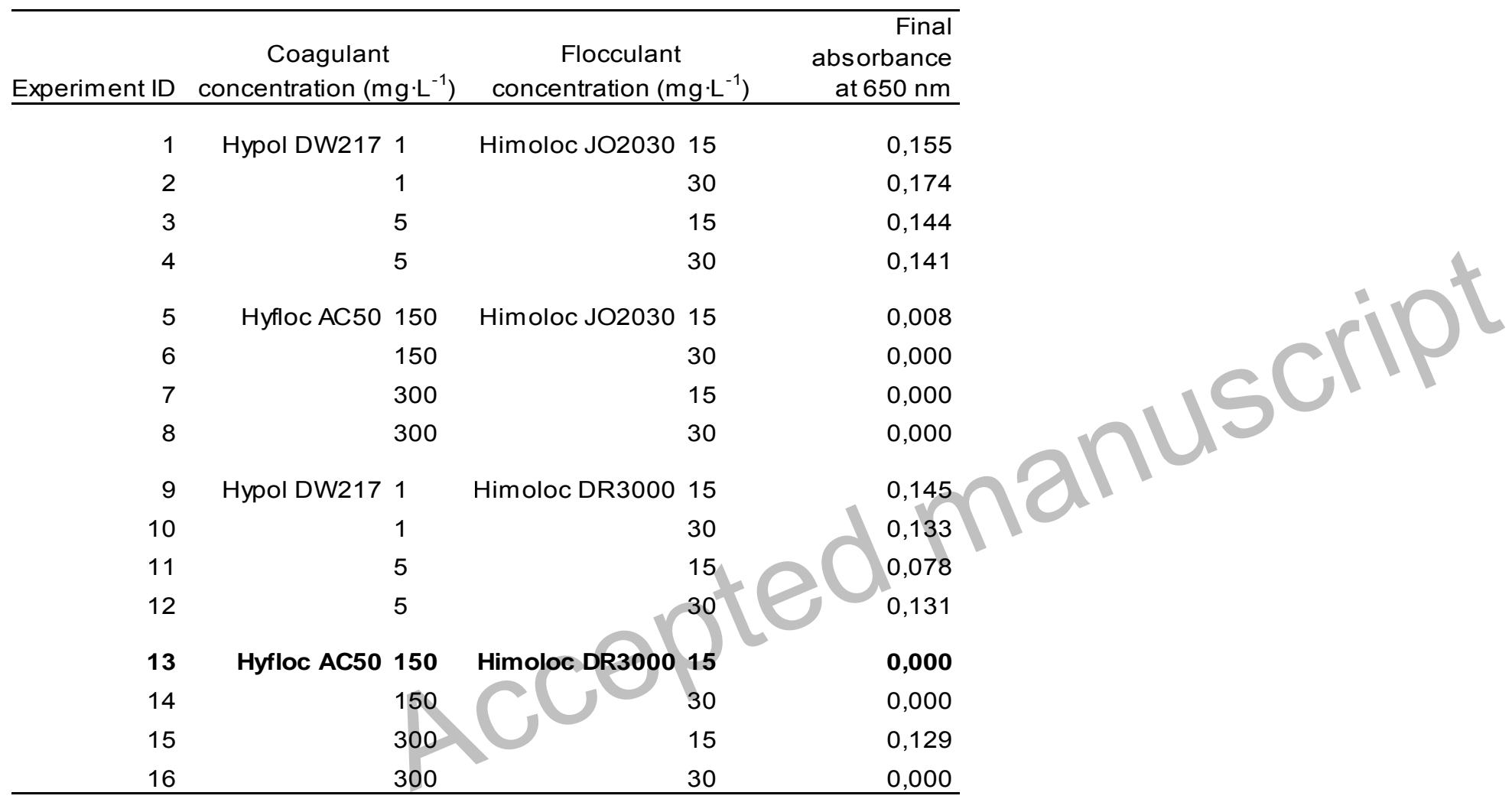


Table 3. Coagulant - flocculant doses used in the different HWW.

\begin{tabular}{rrr}
\hline & $\begin{array}{r}\text { Coagulant } \\
\text { HWW }\end{array}$ & $\begin{array}{r}\text { Flocculant } \\
\text { concentration }\left(\mathrm{mg} \cdot \mathrm{L}^{-1}\right)\end{array}$ \\
\hline 1 & 150 & 15 \\
2 & 95 & 11 \\
3 & 37 & 4,5 \\
\hline
\end{tabular}

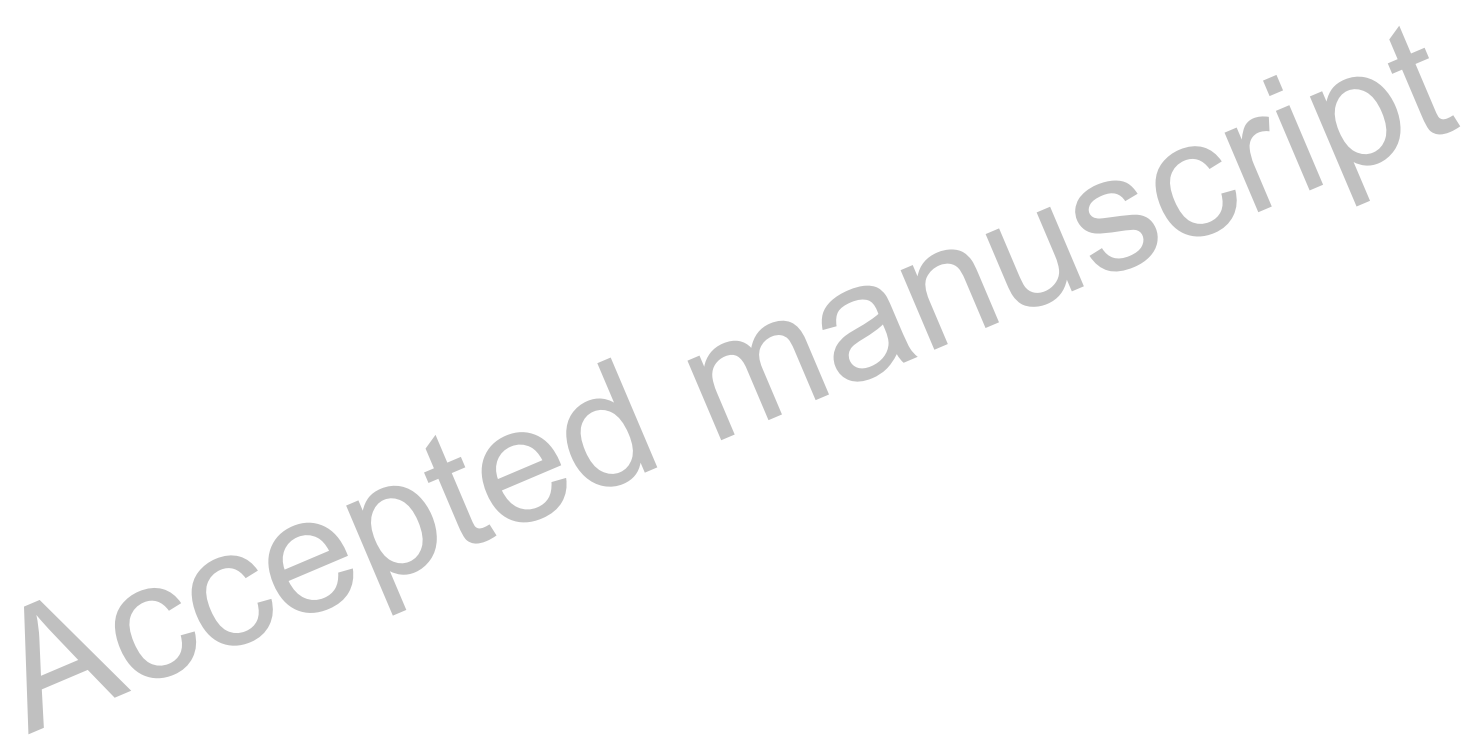

